\title{
PRODUTIVIDADE DE CULTIVARES DE MILHO EM ADAMANTINA, REGIÃO DA NOVA ALTA PAULISTA
}

\author{
SILVA, Lucas de Souza ${ }^{1}$; NAKAYAMA, Fernando Takayuki ${ }^{2}$; SANTOS, Guilherme Xavier Lúcio ${ }^{3}$; \\ CORDEIRO JUNIOR, Paulo Sérgio ; DONÁ, Sérgio ${ }^{5}$; FINOTO, Everton Luis ${ }^{6}$; BRANDÃO, Rebeca \\ Pereira $^{1}$; RODRIGUES, Lucas ${ }^{1}$; VITORINO, Rodrigo ${ }^{7}$
}

ISSUE DOI: $10.3738 / 1982.2278 .3633$

\begin{abstract}
RESUMO: A cultura do milho consolida-se atualmente como a segunda cultura de maior importância para a agricultura brasileira, além de ser o segundo cereal mais consumido mundialmente, devido suas diversas formas de utilização que vão desde a alimentação animal até a indústria de alta tecnologia. O Brasil está entre os maiores produtores, tendo representatividade em todo território nacional. O objetivo do presente trabalho foi avaliar a produtividade de quinze cultivares de milho conduzidos em sistema de plantio direto para a região da Nova Alta Paulista. O experimento foi conduzido no período de outubro de 2018 a fevereiro de 2019, em área experimental pertencente a Agência Paulista de Tecnologia dos Agronegócios - APTA, no município de Adamantina, SP. Foram avaliadas as seguintes variáveis: massa de espiga com palha; massa de espiga; massa de grãos; massa de 100 grãos e número de grãos. De acordo com os resultados obtidos, as maiores produtividades, com valores expressivos comercialmente, foram observados nos híbridos AG 8740, AG 8076, AG 8088, AG3700 e AG8700. Estes cinco híbridos AG expressam alto potencial produtivo, ultrapassando os $10.000 \mathrm{~kg} \mathrm{ha}^{-1}$ e todos os híbridos NX atingem produtividade em torno de $9.000 \mathrm{~kg} \mathrm{ha}^{-1}$. Há necessidade de dar continuidade e se estender o trabalho comparando-se mais cultivares de milho nas condições edafoclimáticas da Alta Paulista.
\end{abstract}

Palavras-Chave: Zea mays. Plantio direto. Variedades. Tecnologia. Hibridos

\section{MAIZE VARIETIES PRODUCTIVITY FOR ADAMANTINA, NOVA ALTA PAULISTA REGION}

\begin{abstract}
SUMMARY: The maize (corn) crop is currently consolidated as the second most important crop for Brazilian agriculture, as well as being the second most consumed cereal worldwide, due to its diverse uses ranging from animal feed to the high technology. Brazil is among the largest producers, with representativeness throughout the country. The objective of the present work was to evaluate the productivity of fifteen maize varieties conducted under no - tillage system for the Nova Alta Paulista region, conducted from October 2018 to February 2019, in an experimental area belonging to APTA - Agência Paulista de Tecnologia dos Agronegócios, in the municipality of Adamantina - SP, in a system of direct sowing analyzing the following variables: Tang pile mass; Spike mass; Grain mass; Weight of 100 grains and number of grains.According to the results, the highest yields, with commercially significant values, were observed in hybrids AG 8740, AG 8076, AG 8088, AG3700 and AG8700. These five AG hybrids express high productive potential, exceeding 10,000 kg ha-1 and all NX hybrids reach productivity around $9,000 \mathrm{~kg}$ ha- 1 . There is a need to continue and extend the work by comparing more maize cultivars under the edaphoclimatic conditions of the Upper Paulista.
\end{abstract}

Keywords: Zea mays. Direct planting. Varieties. Technology. Hybrids.

\footnotetext{
${ }^{1}$ Graduando em Agronomia - Centro Universitário de Adamantina - UNIFAI, Adamantina SP;

${ }^{2}$ Pesquisador Cientifico, Dr. - PRDTA Alta Paulista, Adamantina, SP;

${ }^{3}$ Engenheiro Agrônomo, Bolsista de Aperfeiçoamento Técnico FUNDAG/APTA/SAA - PRDTA Centro Norte, Pindorama, SP;

${ }^{4}$ Mestrando em Produção Vegetal - FCAV-UNESP, Jaboticabal, SP;

${ }^{5}$ Pesquisador Cientifico, Msc - PRDTA Médio Paranapanema, Assis, SP;

${ }^{6}$ Pesquisador Cientifico, Dr. - PRDTA Centro Norte, Pindorama, SP;

${ }^{7}$ Assistente Técnico de Pesquisa - PRDTA Alta Paulista - Adamantina, SP.
} 


\section{INTRODUÇÃO}

A cultura do milho (Zea mays) consolida-se atualmente como a segunda cultura de maior importância para a agricultura brasileira, além de ser o segundo cereal mais consumido mundialmente, devido suas diversas formas de utilização que vão desde a alimentação animal até a indústria de alta tecnologia.

Por outro lado, os prêmios dos portos brasileiros estão em queda, o que desvaloriza a paridade de exportação e, por consequência, impedem que a tendência de baixa das cotações domésticas do milho seja contida. No cenário doméstico, mais uma vez a CONAB aponta um aumento da estimativa de produção de milho, fazendo com que os estoques de passagem do cereal da safra 2018/19 estimados subam para 16,5 milhões de toneladas (CONAB, 2019).

A necessidade de alimentar anualmente uma população maior e sempre em crescimento, leva o homem a uma contínua procura de melhores rendimentos unitários das plantas que lhe servem de subsistência (RUSCHEL, 1970). Atualmente, a cultura do milho consolidou-se no mercado nacional a segunda cultura de maior importância econômica para a agricultura brasileira, além de ser o cereal mais consumido mundialmente devido suas inúmeras formas de comercialização, que parte desde a indústria dos micro processados, até a nutrição animal (EMBRAPA, 2006). A cultura é considerada tradicional para a região Oeste de São Paulo, sobretudo por existir uma alta demanda pelo grão, principalmente para suprir as cadeias produtivas zootécnicas e por ser considerada uma excelente opção para reforma de áreas de pastagens degradadas, o que torna a adoção de sistemas de cultivo como o "Sistema Barreirão" como opção sustentável.

Neste contexto, salienta-se a importância da utilização de materiais genéticos produtivos e adaptados, para garantir a sustentabilidade do sistema. Para essa grande expressão de potencial produtivo inúmeros, fatores são de extrema importância que devem ser levados em conta, sendo primordialmente a escolha do material que irá trabalhar. A gama de materiais disponíveis atualmente no mercado é muito grande.

Contudo, um fator muito importante para a determinação da sua produção é qual a cultivar mais recomendada para a sua região edafoclimática, levando em consideração que muitas variedades podem expressar características diferentes quando submetidas a diferentes regiões, tipos de solo, fotoperiodo e afins, elevando ou não o sua expressão máxima do potencial produtivo, o que pode ocasionar uma grande perda de lavoura.

Outro ponto muito importante para a escolha da cultivar é qual o segmento que mesma terá, ou seja, seu fim de aplicação, saber escolher a cultivar segundo a sua necessidade (silagem, massa de palhada, grãos, etc.) é de fundamental importância para o êxito de sua lavoura. Objetivou-se por meio deste trabalho, avaliar os componentes produtivos de diferentes cultivares de milho para as condições edafoclimáticas da região da Nova Alta Paulista.

\section{MATERIAL E MÉTODO}

O presente estudo foi conduzido na fazenda de pesquisa pertencente à Agência Paulista de Tecnologia dos Agronegócios - APTA, situada no município de Adamantina, SP, que se encontra numa altitude de 453 metros, o clima da região é classificado como AW, segundo Köppen, tropical úmido com pluviosidades mais elevada no verão e seca no inverno. O Solo da região é classificado como Argissolo vermelho/amarelo eutrófico a moderado com textura arenosa / média e apresenta uma topografia ondulada (EMBRAPA, 1999). O período de condução da pesquisa foi novembro de 2018 a fevereiro de 2019, os materiais avaliados foram: AG 8088; AG 8061; AG 8690; AG 7098; AG 3700; AG 8740; AG 8076; AG 
8700; BM 01; BM 02; BM 03; BM 04; NX 468; NX 225 e NX 544, sendo que os materiais NX não possuem tecnologia transgênica.

As características químicas do solo antes da implantação do experimento apresentam os valores descritos na Tabela 1.

Tabela 1. Análise química do solo da área do experimento, Adamantina 2018.

\begin{tabular}{cccccccccccc}
\hline $\begin{array}{c}\mathrm{pH} \\
\left(\mathrm{CaCl}_{2}\right)\end{array}$ & $\begin{array}{c}\mathrm{MO} \\
\left(\mathrm{g} \mathrm{dm}^{-3}\right)\end{array}$ & $\begin{array}{c}\mathrm{P} \\
\mathrm{mg} \mathrm{dm}^{-3}\end{array}$ & $\mathrm{~K}$ & $\mathrm{Ca}$ & $\mathrm{Mg}$ & $\mathrm{H}+\mathrm{Al}$ & $\mathrm{Al}$ & $\mathrm{SB}$ & $\mathrm{T}$ & $\mathrm{V} \%$ & $\mathrm{~m} \%$ \\
\hline & & & & & & & & & & \\
\hline & & & & & & & & \\
\hline
\end{tabular}

SB: Soma das bases; V\%: Saturação por bases; m\%: saturação por alumínio; T: capacidade de troca catiônica

Setenta dias antecedendo a semeadura a saturação por bases foi elevada a $70 \%$ com aplicação de calcário, seguindo recomendação do Boletim Técnico 100 (RAIJ, 1987). A semeadura do experimento ocorreu no dia 22 de outubro de 2018 através do sistema de semeadura direta seguindo o delineamento experimental empregado de blocos ao acaso com densidade populacional de 5,5 por metro linear com espaçamento de 0,90 m entre linhas. Utilizando como adubo de plantio o formulado 08-28-16 seguindo a dosagem recomendada pelo Boletim Técnico 100 (RAIJ, 1987), seguido com uma adubação de cobertura com $160 \mathrm{~kg}$ de ureia 30 dias após a emergência das plantas.

Figura 1. Precipitação e distribuição hídrica para o período experimental de outubro de 2018 a janeiro de 2019.

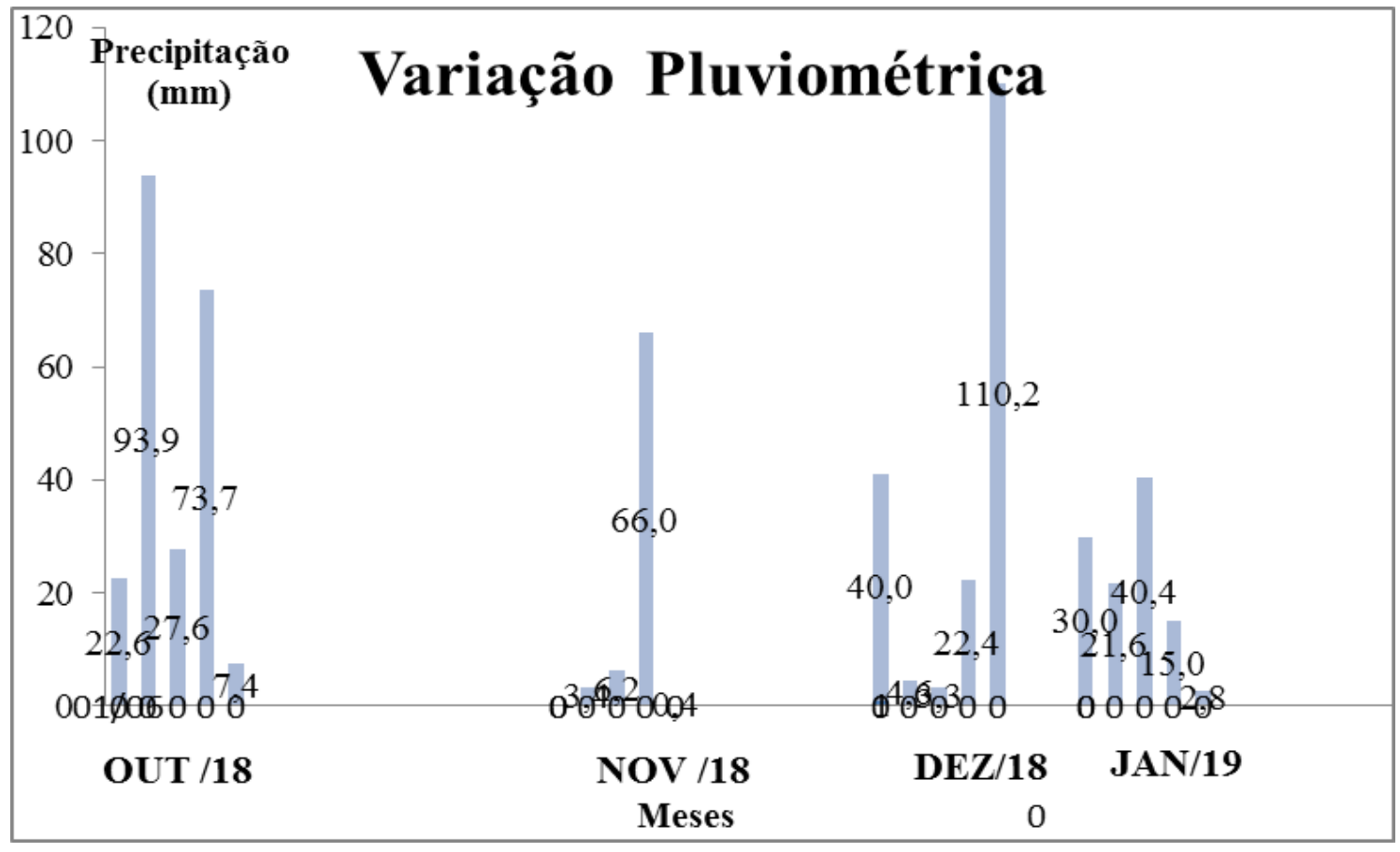

As variáveis analisadas no presente trabalho foram a massa de espiga com palha, massa de espiga, massa de grãos, massa de 100 grãos, número de grãos e produtividade para o ano agrícola 2018/2019. Para avaliação foram colhidas 10 plantas contendo uma única espiga com quatro repetições por parcela, totalizando 40 espigas de cada material. Foram pesadas separadamente cada variável utilizando 
uma balança digital de pêndulo e balança digital de mesa, os materiais após debulhados foram separados em sacos de papel com umidade corrigida para 13\% para determinação de grãos por espiga, massa de 100 grãos e produtividade.

Após o termino das avaliações, os dados obtidos foram submetidos a análise de variância através do teste $\mathrm{F}$ e quando significativos comparados pelo teste Tukey a 5\% de probabilidade.

\section{RESULTADO E DISCUSSÃO}

A safra 2018/19 foi marcada por apresentar distribuição hídrica irregular durante o desenvolvimento da cultura (Figura 01), o que proporcionou um déficit no período vegetativo e também reprodutivo. Intervalos de mais de 20 dias sem chuva atrelado a alta temperatura, durante os meses de dezembro e janeiro prejudicaram a safra. Situação semelhante foi encontrada por Garbuglio et al. (2018) no ano agrícola de 2017/2018, no qual também afirmaram que o baixo índice pluviométrico nas fases iniciais da cultura também foi um fator que alterou significativamente a produtividade média, o que girou em torno de $8.745 \mathrm{~kg} \mathrm{ha}^{-1}$. Na região da Nova Alta Paulista no ano agrícola de 2018/2019, a produção final com as variáveis de cultivares, girou em média de $9.021,43 \mathrm{~kg} \mathrm{ha}^{-1}$, apesar da diferença entre as regiões edafoclimáticas, tornou-se possível a conclusão de que uma anormalidade de precipitação pode ocasionar uma grande perda de produtividade de determinadas cultivares principalmente quando ocorridas nas fases iniciais da cultura, florescimento e enchimento de grãos.

Os resultados referentes a valores médios de massa de espiga com palha, sem palha e massa de grãos estão apresentados na tabela 02. Para massa de espiga com palha o material AG3700 obteve os maiores valores, diferindo significativamente de AG 8061, AG 8690, BM 01, BM 02, BM 03, BM 04 e NX 468. Partindo para as análises da variável de milho por e espiga os materiais AG 8740 e AG 8076 apresentaram maiores resultados de massa de grãos, o que tornou possível a observação de que determinados materiais possuem significativamente maior massa de sabugo e palha do que massa de grãos, tais como os materiais AG 8061, AG 8690, AG 7098, BM 01, BM 02, BM 03, BM 04, NX 468 e NX 225.

Tabela 2. Valores médios de massa de espiga com palha $(\mathrm{kg})$, massa de espiga $(\mathrm{kg})$ e massa de grãos $(\mathrm{kg})$, (umidade 13\%) obtidos em espigas de milho conduzidos em sistema de plantio direto, Adamantina, SP, 2019.

(Continua)

\begin{tabular}{llll}
\hline $\begin{array}{c}\text { Avaliações/ } \\
\text { Cultivares }\end{array}$ & $\begin{array}{c}\text { Massa de espiga com } \\
\text { palha }\end{array}$ & Massa de espiga & \multicolumn{1}{c}{$\begin{array}{c}\text { Massa de grãos por } \\
\text { espiga }\end{array}$} \\
\hline AG 8740 & $0,27 \mathrm{ab}$ & $0,24 \mathrm{abc}$ & $0,19 \mathrm{a}$ \\
AG 8076 & $0,26 \mathrm{abcd}$ & $0,24 \mathrm{ab}$ & $0,19 \mathrm{a}$ \\
AG 3700 & $0,28 \mathrm{a}$ & $0,25 \mathrm{a}$ & $0,19 \mathrm{ab}$ \\
AG 8700 & $0,25 \mathrm{abcd}$ & $0,24 \mathrm{abc}$ & $0,19 \mathrm{abc}$ \\
AG 8088 & $0,27 \mathrm{abc}$ & $0,24 \mathrm{abc}$ & $0,18 \mathrm{abcd}$ \\
AG 8700 & $0,25 \mathrm{abcd}$ & $0,24 \mathrm{abc}$ & $0,19 \mathrm{abc}$ \\
NX 225 & $0,26 \mathrm{abc}$ & $0,22 \mathrm{abcd}$ & $0,17 \mathrm{bcde}$ \\
NX 544 & $0,24 \mathrm{abcde}$ & $0,21 \mathrm{abcde}$ & $0,16 \mathrm{bcdef}$ \\
NX 468 & $0,21 \mathrm{bcde}$ & $0,19 \mathrm{bcde}$ & $0,16 \mathrm{bcdef}$ \\
AG 7098 & $0,23 \mathrm{abcde}$ & $0,21 \mathrm{abcde}$ & $0,15 \mathrm{cdef}$
\end{tabular}


Tabela 2. Valores médios de massa de espiga com palha ( $\mathrm{kg})$, massa de espiga $(\mathrm{kg})$ e massa de grãos $(\mathrm{kg})$, (umidade 13\%) obtidos em espigas de milho conduzidos em sistema de plantio direto, Adamantina, SP, 2019.

(Conclusão)

\begin{tabular}{llll}
\hline $\begin{array}{c}\text { Avaliações/ } \\
\text { Cultivares }\end{array}$ & \multicolumn{1}{c}{$\begin{array}{c}\text { Massa de espiga com } \\
\text { palha }\end{array}$} & Massa de espiga & $\begin{array}{c}\text { Massa de grãos por } \\
\text { espiga }\end{array}$ \\
\hline AG 8061 & $0,21 \mathrm{cde}$ & $0,19 \mathrm{cde}$ & $0,15 \mathrm{cdef}$ \\
BM 03 & $0,20 \mathrm{de}$ & $0,19 \mathrm{de}$ & $0,15 \mathrm{def}$ \\
BM 02 & $0,21 \mathrm{cde}$ & $0,20 \mathrm{bcde}$ & $0,15 \mathrm{ef}$ \\
AG 8690 & $0,21 \mathrm{cde}$ & $0,19 \mathrm{cde}$ & $0,14 \mathrm{ef}$ \\
BM 01 & $0,20 \mathrm{de}$ & $0,18 \mathrm{de}$ & $0,14 \mathrm{ef}$ \\
\hline Teste F & 6,81 & $7,03^{* *}$ & $9,69^{* *}$ \\
CV\% & 9,79 & 9,13 & 8,24 \\
DMS & 0,05 & 0,05 & 0,03
\end{tabular}

As médias seguidas pela mesma letra minúscula na coluna não diferem estatisticamente entre si pelo Teste de Tukey ao nível de 5\% de probabilidade.

Tabela 3. Valores médios de massa de 100 grãos (gramas), número médio de grãos por espiga e produtividade (umidade 13\%), obtidos em espigas de milho conduzidos em sistema de plantio direto, Adamantina, SP, 2019.

\begin{tabular}{|c|c|c|c|}
\hline $\begin{array}{l}\text { Avaliações/ } \\
\text { Cultivares }\end{array}$ & $\begin{array}{l}\text { Massa de } 100 \text { grãos } \\
(\mathrm{g})\end{array}$ & $\begin{array}{l}\text { Número médio de } \\
\text { grãos por espiga }\end{array}$ & $\begin{array}{c}\text { Produtividade } \\
\mathrm{Kg} \mathrm{ha}^{-1}\end{array}$ \\
\hline AG 8740 & $32,00 \mathrm{bcd}$ & 564,00 abcde & $10.749,89 \mathrm{a}$ \\
\hline AG 8076 & 30,00 cde & $641,50 \mathrm{a}$ & $10.740,19 \mathrm{a}$ \\
\hline AG 3700 & $34,50 \mathrm{abc}$ & $602,00 \mathrm{abc}$ & $10.536,00 \mathrm{ab}$ \\
\hline AG 8700 & $40,00 \mathrm{a}$ & 526,00 bcdef & $10.434,61 \mathrm{abc}$ \\
\hline AG 8088 & $37,75 \mathrm{ab}$ & $657,50 \mathrm{a}$ & $10.025,91 \mathrm{abcd}$ \\
\hline NX 225 & 30,50 cde & 580,50 abcd & $9.040,20$ abcde \\
\hline NX 544 & $27,00 \mathrm{de}$ & $636,50 \mathrm{a}$ & $8.851,30$ abcde \\
\hline NX 468 & $35,50 \mathrm{abc}$ & 532,00 bcdef & $8.761,02$ abcde \\
\hline AG 7098 & $38,75 \mathrm{a}$ & 475,50 ef & $8.393,68$ bcde \\
\hline BM 03 & 30,75 cde & 518,50 cdef & $8.305,47$ cde \\
\hline BM 02 & $37,24 \mathrm{ab}$ & $489,50 \mathrm{def}$ & $8.174,92$ cde \\
\hline AG 8061 & $39,25 \mathrm{a}$ & $441,00 \mathrm{f}$ & $8.048,73 \mathrm{de}$ \\
\hline AG 8690 & $34,25 \mathrm{abc}$ & 516,00 cdef & $8.029,08 \mathrm{de}$ \\
\hline BM 01 & $26,00 \mathrm{e}$ & $625,50 \mathrm{ab}$ & $7.795,75 \mathrm{e}$ \\
\hline BM 04 & $27,25 \mathrm{de}$ & 575,50 abcde & $7.434,64 \mathrm{e}$ \\
\hline Teste F & $16,21 * *$ & $10,98^{* *}$ & $7,59 * *$ \\
\hline $\mathrm{CV} \%$ & 6,99 & 7,11 & 9,35 \\
\hline DMS & 5,94 & 101,11 & $2.148,25$ \\
\hline
\end{tabular}

As médias seguidas pela mesma letra minúscula na coluna não diferem estatisticamente entre pelo Teste de Tukey ao nível de $5 \%$ de probabilidade. 
Para produtividade, observa-se que os as maiores produtividades, com valores expressivos comercialmente, foram obtidos nos híbridos AG 8740, AG 8076, AG 8088, AG3700 e AG8700. Estes cinco híbridos expressam alto potencial produtivo, ultrapassando os $10.000 \mathrm{~kg} \mathrm{ha}^{-1}$, o equivalente a mais de 160 sacas, apresentando mais de 3 mil quilos em relação a variedade BM 04 com a menor produtividade. Todos os híbridos NX atingem produtividade em torno de $9.000 \mathrm{~kg} \mathrm{ha}^{-1}$. A produtividade do híbridos AG, anteriormente mencionados, diferiram significativamente dos materiais AG 8061, AG 8690, AG 7098, BM 01, BM 02, BM 03 e BM 04 que apresentaram resultados de produtividade em torno de $8.000 \mathrm{~kg} \mathrm{ha}^{-1}$. Vale a pena salientar que os materiais NX 468, NX 225 e NX 544, não apresentam nenhum tipo de tecnologia transgênica inserida no grão, enquanto os demais materiais possuem tecnologia PRO2 e PRO3.

De acordo com os resultados encontrados no presente trabalho considera-se que devido as condições edafoclimáticas e a irregularidade na distribuição pluviométrica durante o ano agrícola que se desenvolveu a pesquisa, as cultivares que mais apresentaram adaptação e vigor vegetativo para esse tipo de situação se diferiram significativamente das demais cultivares implantadas no experimento, elevando sua produção a mais de 3 mil kg ha- ${ }^{1}$ em relação a outras variedades, em termos de massa de grãos e produtividade final, o que torna interessante para os produtores da região da Nova Alta Paulista a escolha correta de materiais mais resistentes as secas.

\section{CONCLUSÃO}

Conclui-se que cinco dos híbridos AG expressam alto potencial produtivo, ultrapassando os $10.000 \mathrm{~kg} \mathrm{ha}^{-1}$ e todos os híbridos NX atingem produtividade em torno de $9.000 \mathrm{~kg} \mathrm{ha}^{-1}$.

Há necessidade de dar continuidade e se estender o trabalho comparando-se outros cultivares de milho disponíveis no mercado nas condições edafoclimáticas da Alta Paulista.

\section{REFERÊNCIAS}

CONAB - Companhia Nacional de Abastecimento Agricola. ACOMPANHAMENTO DA SAFRA

BRASILEIRA DE GRÃOS. v. 6 - Safra 2018/19, n.8 - Oitavo levantamento, maio 2019.

EMPRESA BRASILEIRA DE PESQUISA AGROPECUÁRIA (EMBRAPA) Sistema Brasileiro de classificação de Solos. Centro Nacional de Pesquisa de Solos. Rio de Janeiro, 1999, 0412p.

EMPRESA BRASILEIRA DE PESQUISA AGROPECUÁRIA (EMBRAPA) Aspectos Físicos, Químicos e Tecnológicos do Grão de Milho. p. 6, Sete Lagoas, MG Dezembro, 2006.

RAIJ, B. VAN; CANTARELLA, H.; QUAGGIO, J.A.; FURLANI, A.M.C. Recomendações de adubação e calagem para o Estado de São Paulo. 2.ed. Campinas: IAC, 1997. 285p. (Boletim Técnico, 100).

RUSCHEL, R. Análise da produtividade dos cultivares sintético e híbridos de milho. Pesquisa Agropecuária Brasileira, v. 5, n.1, p. 345-350, 1970.

GARBUGLIO, D. D.; SHIOGA, P. S.; GERAGE, A. C.; ARAUJO, P. M.; BIANCO, R.; CUSTODIO, A. A. P.; BARROS, A. S. R. Avaliação estadual de cultivares de milho Safra 2017/2018. Londrina: INTITUTO AGRONOMICO DO PARANÁ (IAPAR), 2018. 59 p. il. (IAPAR. Boletim técnico, 91) 